Supporting Information

\title{
Aqueous Suspensions of Cellulose Oligomer Nanoribbons for Growth and Natural Filtration-Based Separation of Cancer Spheroids
}

Takeshi Serizawa, ${ }^{*}{ }^{\dagger}$ Tohru Maeda, ${ }^{\dagger}$ Saeko Yamaguchi,${ }^{\dagger}$ and Toshiki Sawada ${ }^{\dagger, *}$

$\dagger$ Department of Chemical Science and Engineering, School of Materials and Chemical Technology, Tokyo Institute of Technology, 2-12-1 Ookayama, Meguro-ku, Tokyo 1528550, Japan

$\$$ Precursory Research for Embryonic Science and Technology (PRESTO), Japan Science and Technology Agency (JST), 4-1-8 Honcho, Kawaguchi-shi, Saitama 332-0012, Japan

*E-mail: serizawa@mac.titech.ac.jp 


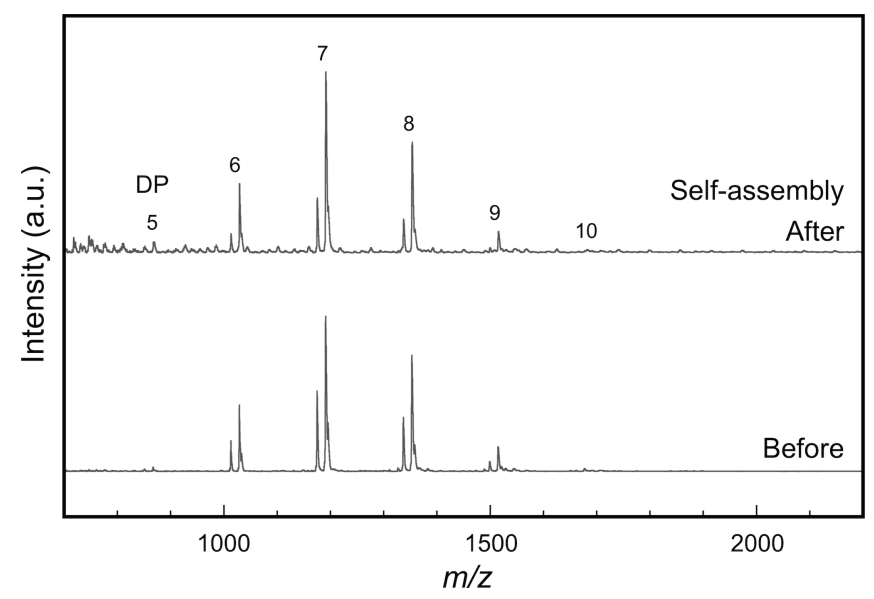

Figure S1. Matrix-assisted laser desorption/ionization time-of-flight mass spectrum of the present cellulose oligomers synthesized according to our previous study. ${ }^{1}$

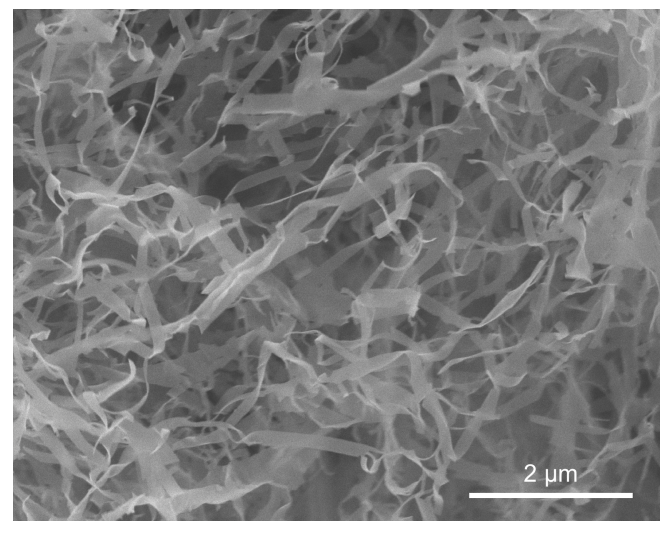

Figure S2. Scanning electron microscopy image of the cellulose oligomer hydrogels composed of crystalline nanoribbon networks, which were prepared in our previous study. $^{2}$ Copyright 2020 American Chemical Society.

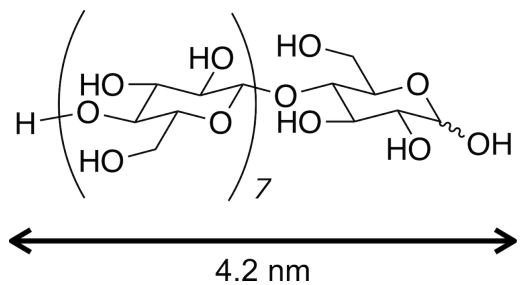

Figure S3. The estimated molecular length of cellooctaose, which was estimated from the structure of the cellulose II allomorph. ${ }^{3}$ 


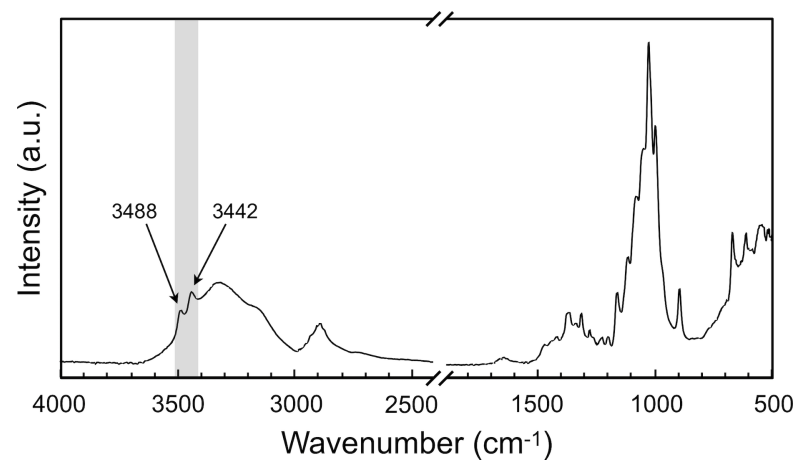

Figure S4. ATR-FTIR spectrum of crystalline nanoribbons composed of cellulose oligomers. Two characteristic peaks at 3488 and $3442 \mathrm{~cm}^{-1}$ were assigned to the $\mathrm{OH}$ stretching vibration bands of intrachain hydrogen-bonded hydroxyl groups in the cellulose II allomorph. ${ }^{4}$

(a)

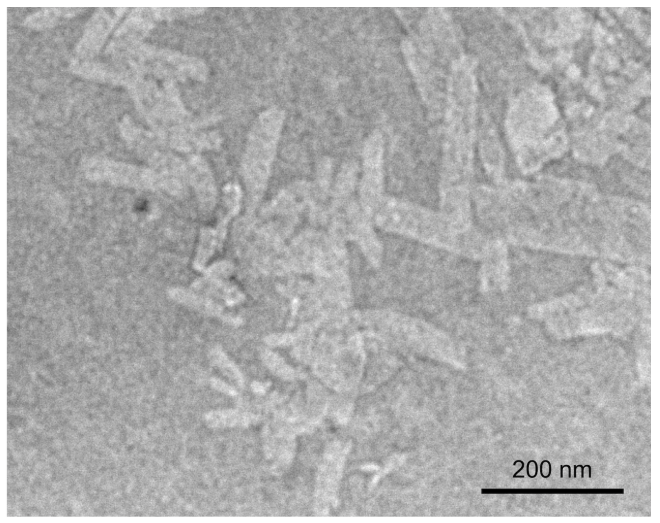

(b)

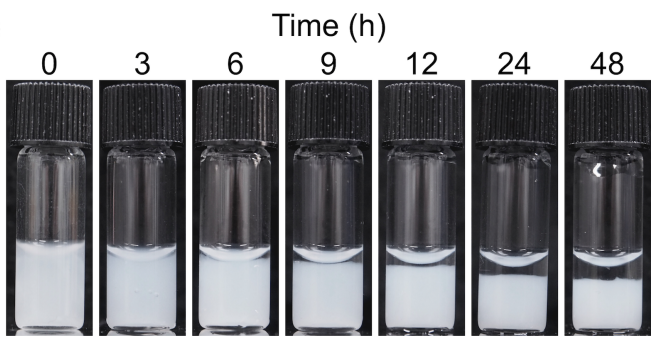

Figure S5. (a) TEM image of sonication-treated nanoribbons and (b) stability of the suspensions in ultrapure water at a nanoribbon concentration of $0.50 \%(\mathrm{w} / \mathrm{v})$ at $37^{\circ} \mathrm{C}$. 

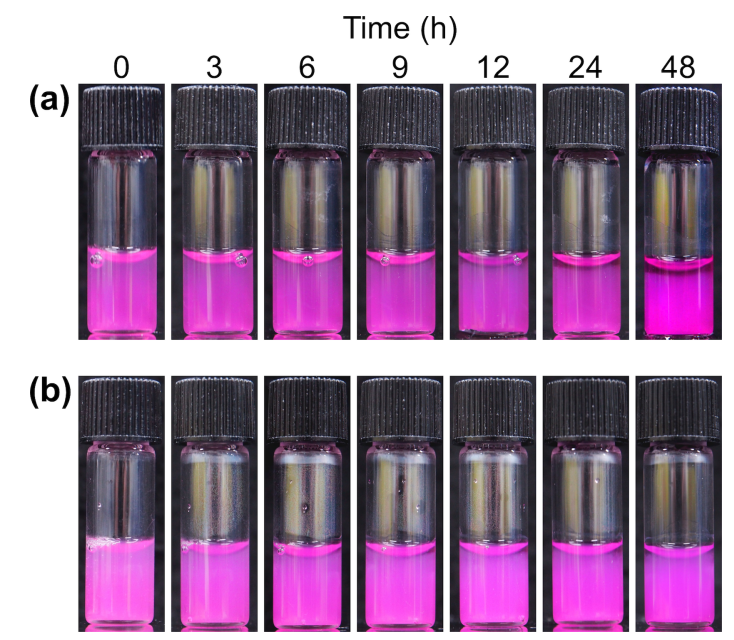

Figure S6. Stability of the nanoribbon suspensions in cell culture media at nanoribbon concentrations of (a) 0.25 and (b) $0.50 \%(\mathrm{w} / \mathrm{v})$ at $37^{\circ} \mathrm{C}$.

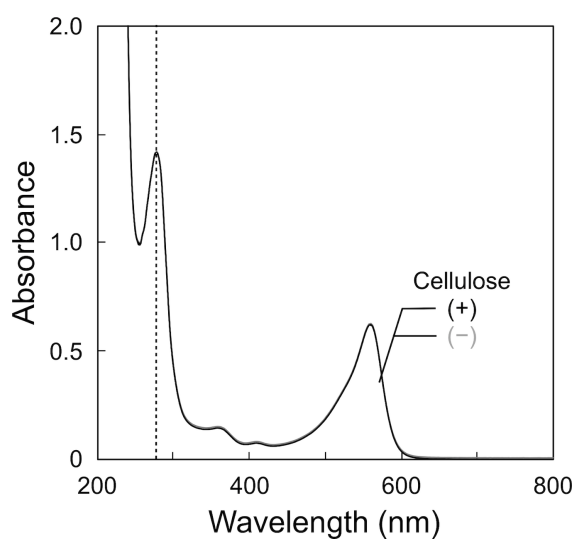

Figure S7. UV-vis absorption spectra of cell culture media before and after incubation with cellulose oligomer nanoribbons at a nanoribbon concentration of $0.50 \%(\mathrm{w} / \mathrm{v})$ at $37{ }^{\circ} \mathrm{C}$ for $1 \mathrm{~h}$. The absorption at around $280 \mathrm{~nm}$ (dotted line) is mainly derived from proteins. 
(a)

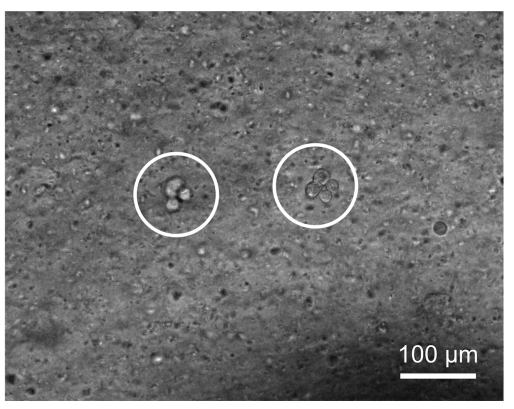

(b)

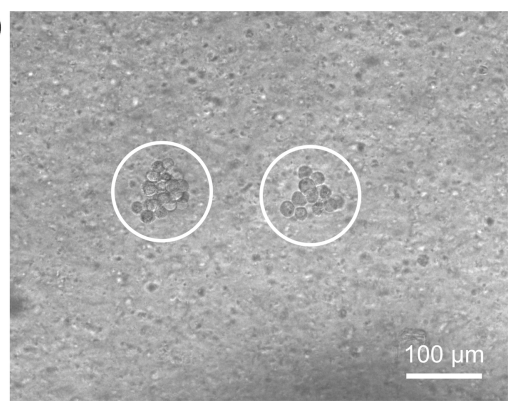

Figure S8. Optical microscopy-based fixed-point observations of HeLa cells cultured at a nanoribbon concentration of $0.50 \%(w / v)$ for (a) $2 \mathrm{~d}$ and (b) $4 \mathrm{~d}$.

(a)

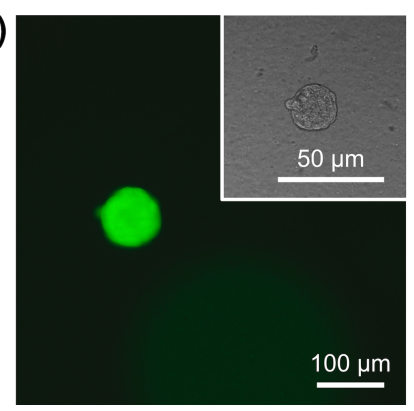

(b)

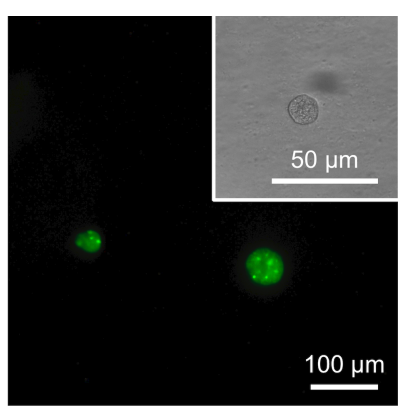

Figure S9. Fluorescence microscopy images of (a) HEK293 and (b) A549 cells cultured at a nanoribbon concentration of $0.50 \%(\mathrm{w} / \mathrm{v})$ for 5 and $7 \mathrm{~d}$, respectively. The insets show optical microscopy images of the cells.

(a)

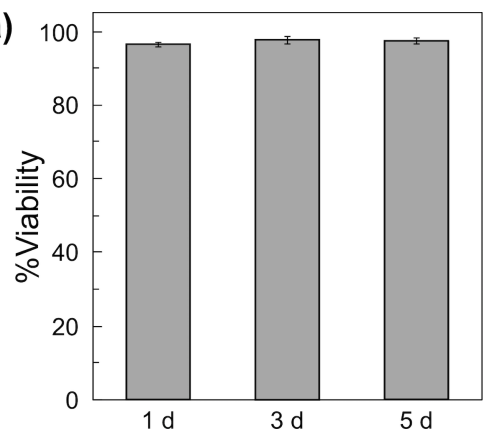

(b)

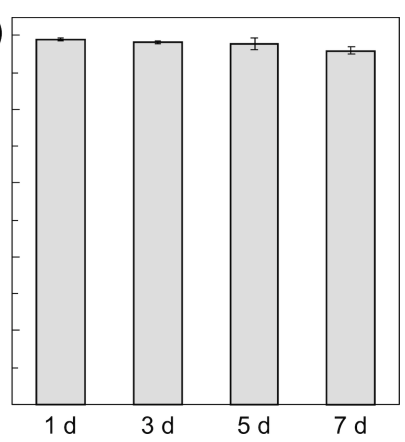

Figure S10. Viability of (a) HEK293 and (b) A549 cells cultured at a nanoribbon concentration of $0.50 \%(\mathrm{w} / \mathrm{v})$ up to 5 and $7 \mathrm{~d}$, respectively. 

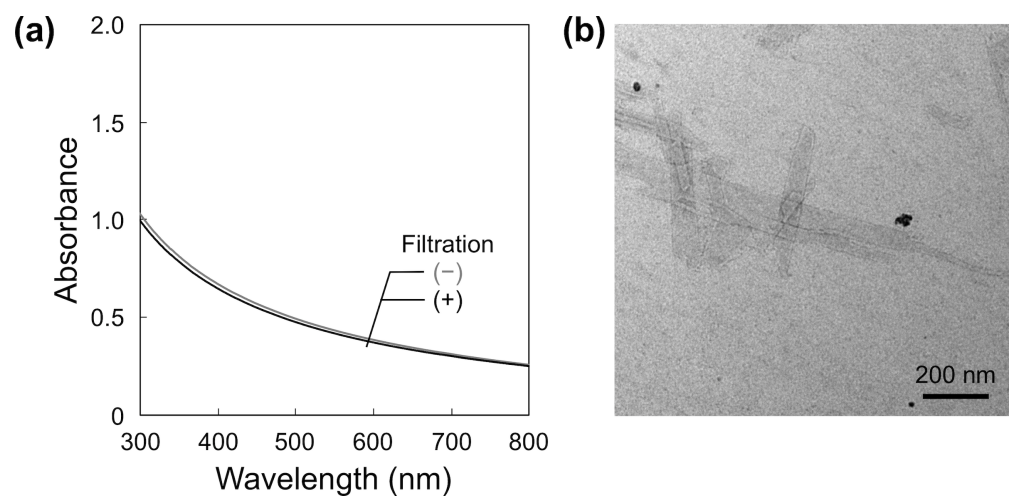

Figure S11. (a) UV-vis absorption spectra of the nanoribbon suspensions before and after natural filtration and (b) TEM image of the cellulose oligomer nanoribbons after natural filtration.

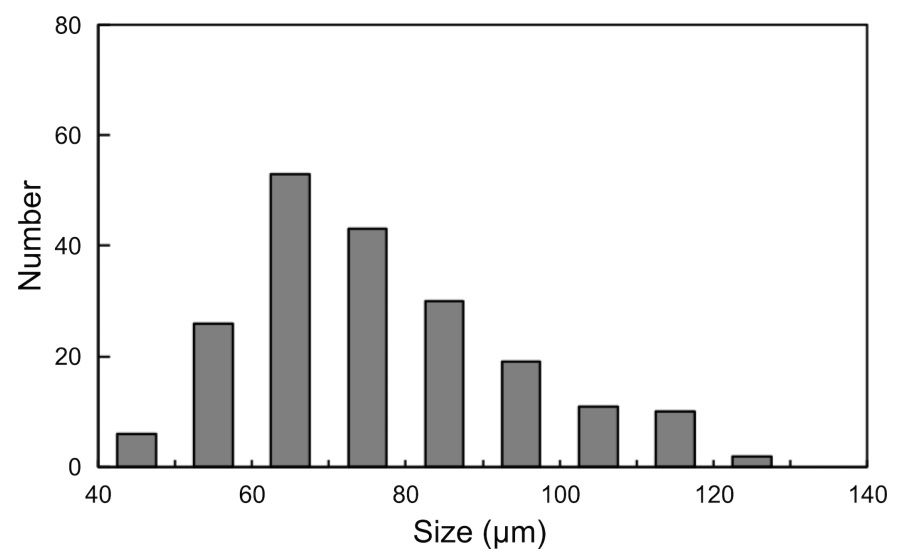

Figure S12. Histogram of frequencies for the diameters of CSs (HeLa cells) separated from the hydrogels composed of nanoribbon networks ${ }^{2}$ at a nanoribbon concentration of $0.50 \%(\mathrm{w} / \mathrm{v})$ for $5 \mathrm{~d}$.

(a)

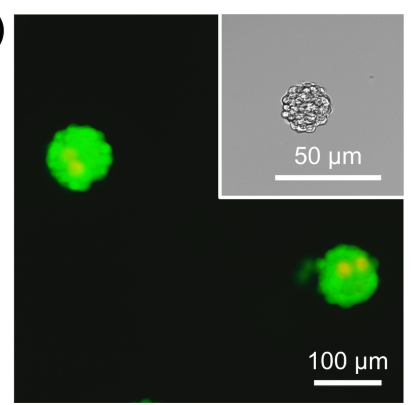

(b)

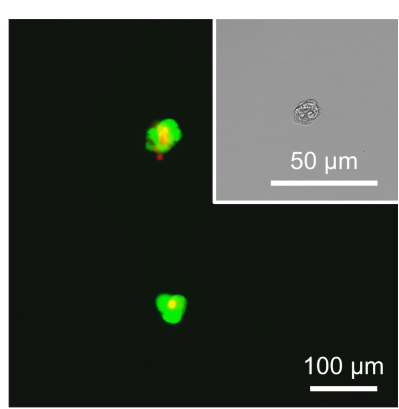

Figure S13. Fluorescence microscopy images of the separated CSs of (a) HEK293 and (b) A549 cells cultured at a nanoribbon concentration of $0.50 \%(\mathrm{w} / \mathrm{v})$ for 5 and $7 \mathrm{~d}$, respectively. The insets show optical microscopy images of the CSs. 


\section{REFERENCES}

(1) Serizawa, T.; Fukaya, Y.; Sawada, T. Self-Assembly of Cellulose Oligomers into Nanoribbon Network Structures Based on Kinetic Control of Enzymatic Oligomerization. Langmuir 2017, 33, 13415-13422.

(2) Serizawa, T.; Maeda, T.; Sawada, T. Neutralization-Induced Self-Assembly of Cellulose Oligomers into Antibiofouling Crystalline Nanoribbon Networks in Complex Mixtures. ACS Macro Lett. 2020, 9, 301-305.

(3) Hiraishi, M.; Igarashi, K.; Kimura, S.; Wada, M.; Kitaoka, M.; Samejima, M. Synthesis of Highly Ordered Cellulose II in Vitro Using Cellodextrin Phosphorylase. Carbohydr. Res. 2009, 344, 2468-2473.

(4) Široký, J.; Blackburn, R. S.; Bechtold, T.; Taylor, J.; White, P. Attenuated Total Reflectance Fourier-Transform Infrared Spectroscopy Analysis of Crystallinity Changes in Lyocell Following Continuous Treatment with Sodium Hydroxide. Cellulose 2010, 17, 103-115. 